\title{
Análise da Equalização Transversal Assimétrica em Sistemas QAM
}

\author{
João Célio Brandão e Álvaro Luis Brito
}

\begin{abstract}
Neste trabalho é feita uma análise do esquema de equalização em banda básica, denominado equalização assimétrica. onde os equalizadores das componentes em fase e em quadratura de um sinal QAM não são necessariamente iguais. Partindo da hipótese de que as componentes em fase e em quadratura do sinal QAM apresentam diferença na forma dos pulsos. são desen vol idas expressões para os valores ótimos dos coeficientes de equalizadores assimétricos de comprimento finito segundo o critério do erro médio quadrático. Obtém-se também uma expressão para o erro médio quadrático nínimo e mostra-se que os resultados obtidos recaem nos resultados já conhecidos da equalização simétrica, quando o sinal QAM apresenta sua forma ideal. Finalmente. as expressões desenvol vidas são aplicadas para comprovar. em algumas situações típicas de um sistena rádio digital. a vantagemem em se usar a equalização assimétrica, embora se mostre, também, que esta forma de equalização apresenta convergência mais lenta do que a equalização simétrica.
\end{abstract}

\section{Introdução}

A equalização transversal em sistemas de transmissão digital é uma técnica amplamente utilizada atualmente e cuja teoria básica está bem estabelecida [1]. Para sistemas com modulação de amplitude em quadratura, a estrutura usual consiste de 4 equalizadores transversais como mostrado na Fig.1, onde $\mathrm{x}_{1}(\mathrm{t})$ e $\mathrm{x}_{2}(\mathrm{t})$ são as componentes em fase e em quadratura do sinal digital no receptor e $\mathbf{W}_{\mathrm{i}}=\left(\mathrm{W}_{\mathrm{i},-\mathrm{N}}, \mathrm{W}_{\mathrm{i},-\mathrm{N}+\mathrm{l}}, \ldots, \mathrm{W}_{\mathrm{i}, \mathrm{N}}\right), \mathrm{i}=11,12,21$ e 22, representa um vetor de coeficientes de um equalizador transversal representado na Fig.2.

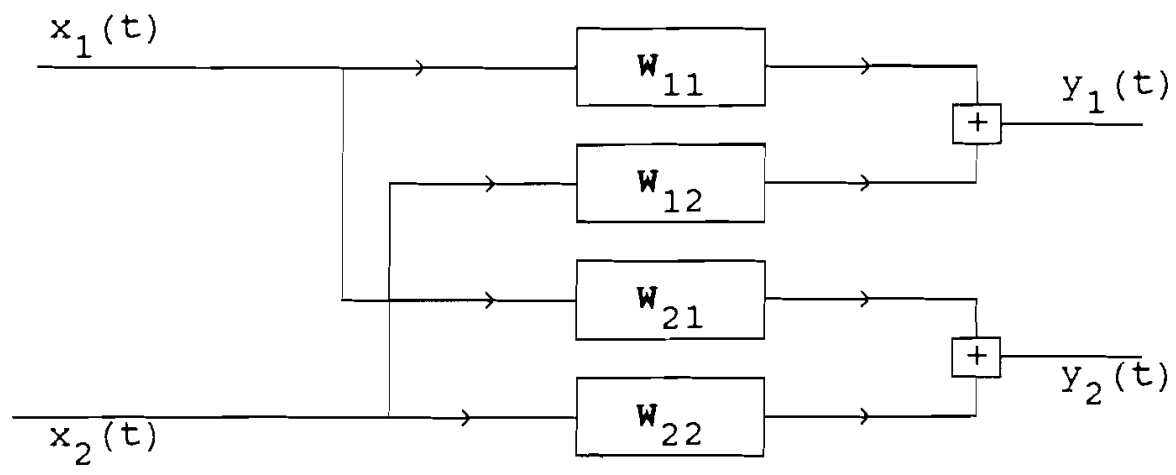

Figura 1 - Estrutura de equalizaçăo assimétrica em banda básica 


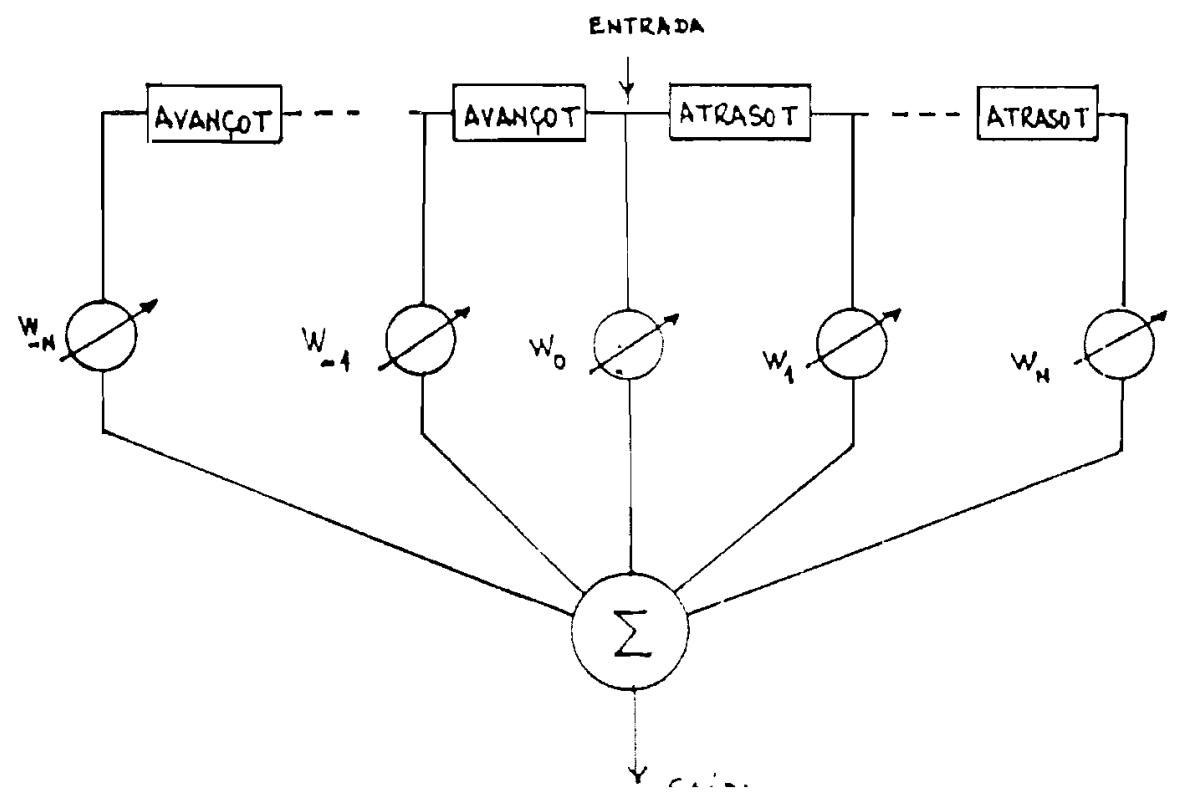

Figura 2 - Equalizador Transversal

Nos esquemas de equalização em banda básica usualmente analisados na literatura, os equalizadores são iguais, dois a dois. Esta igualdade decorre. naturalmente, como solução ótima para a estrutura de equalização de um sinal Q.A \ cuja expressão geral é

$$
x(t)=x_{1}(t) \cos \left(\omega_{c} t\right)-x_{2}(t) \operatorname{sen}\left(\omega_{c} t\right)
$$

Sendo $\mathrm{x}_{1}(\mathrm{t})$ e $\mathrm{x}_{2}(\mathrm{t})$ as componentes em fase e em quadratura dadas por

$$
\mathrm{x}_{1}(\mathrm{t})=\sum_{\mathrm{k}=-\infty}^{\infty} \mathrm{a}_{1 . k} \mathrm{~g}(\mathrm{t}-\mathrm{kT})
$$

e

$$
\mathrm{x}_{2}(\mathrm{t})=\sum_{\mathrm{k}=-\infty}^{\infty} \mathrm{a}_{2, \mathrm{k}} \mathrm{g}(\mathrm{t}-\mathrm{kT})
$$

onde $a_{1, k}$ e $a_{2, k}$ são as amplitudes transmitidas, $g(t)$ é um pulso de baixa frequiência, aqui denominado de pulso básico, e $\omega_{\mathcal{c}}$ é a frequiência da portadora.

6 
A estrutura da Fig.1 com equalizadores iguais dois a dois é equivalente a uma filtra:-.... do sinal $x(t)$ por um filtro passa-faixa de resposta ao impulso $h_{e}(t)$, seguida de demodi:ção coerente para a obtenção das componentes em fase e em quadratura como se mosir seguir.

Chamando de $\tilde{x}(t)$ e $\tilde{h}_{\mathrm{e}}(t)$ as envoltórias complexas de $\mathrm{x}(\mathrm{t})$ e $\mathrm{h}_{\mathrm{e}}(\mathrm{t})$, respectivamente. temse que a envoltória complexa do sinal filtrado, $\widetilde{y}(\mathrm{t})$ será

$$
\widetilde{y}(t)=\frac{1}{2} \widetilde{x}(t) * \tilde{h}_{e}(t)
$$

e as componentes em fase e em quadratura de $y(t)$ serão dadas pelas partes real e imaginária de (3). Desenvolvendo-se (3) tem-se então, para a componente em fase

$$
\mathrm{y}_{1}(\mathrm{t})=\operatorname{Re}[\widetilde{\mathrm{y}}(\mathrm{t})]=\frac{1}{2}\left\{\operatorname{Re}[\widetilde{\mathrm{x}}(\mathrm{t})] * \operatorname{Re}\left[\tilde{\mathrm{h}}_{\mathrm{e}}(\mathrm{t})\right]-\operatorname{Im}[\widetilde{\mathrm{x}}(\mathrm{t})] * \operatorname{Im}\left[\tilde{\mathrm{h}}_{\mathrm{e}}(\mathrm{t})\right]\right\}
$$

e para a componente em quadratura

$$
\mathrm{y}_{2}(\mathrm{t})=\operatorname{Im}[\tilde{\mathrm{y}}(\mathrm{t})]=\frac{1}{2}\left\{\operatorname{Re}[\tilde{\mathrm{x}}(\mathrm{t})] * \operatorname{Im}\left[\tilde{\mathrm{h}}_{\mathrm{e}}(\mathrm{t})\right]+\operatorname{Im}[\tilde{\mathrm{x}}(\mathrm{t})] * \operatorname{Re}\left[\tilde{\mathrm{h}}_{\mathrm{e}}(\mathrm{t})\right]\right\}
$$

Notando que $\operatorname{Re}[\tilde{x}(t)]=x_{1}(t)$ e $\operatorname{Im}[\tilde{x}(t)]=x_{2}(t)$ e confrontando as equações (4a) e (4b) com a estrutura da Fig.1, obser va-se que o mesmo sinal de saída pode ser obtido se os equalizador es com vetor es de coeficientes $\mathbf{W}_{11}$ e $\mathbf{W}_{22}$ corresponderem a $\operatorname{Re}\left[\tilde{\mathrm{h}}_{\mathrm{e}}\right]$, e os equalizadores com vetores de coeficientes $W_{12}$ e $W_{21}$ a $-\operatorname{Im}\left[\widetilde{h}_{\mathrm{e}}\right]$ e $+\operatorname{Im}\left[\tilde{\mathrm{h}}_{\mathrm{e}}\right]$, respectivamente. A equivalência que se acabou de mostrar significa, entre outras coisas, que a equalização transversal em banda básica com os equalizadores iguais, dois a dois, pode também ser realizada em F.I. usando-se apenas dois filtros tranversais.

A existência de imperfeições no modulador e no receptor pode introduzir assimetrias nas componentes em fase e em quadratura do sinal que podem degradar o desempenho, sobretudo de sistemas de modulação com um grande número de níveis. Isto se aplica aos recentes sistemas rádio digitais com modulação QAM-64, QAM-128, QAM-256, etc., onde a equalização convencional tem sido usada normalmente [2,3]. Com a hipótese de assimetria nas componentes em fase e em quadratura, as expressões gerais destas componentes passam a ser

$$
\mathrm{x}_{1}(\mathrm{t})=\sum_{\mathrm{k}=-\infty}^{\infty} \mathrm{a}_{1, \mathrm{k}} \mathrm{g}_{1}(\mathrm{t}-\mathrm{kT})
$$

Revista da Sociedade Brasileira de Telecomunicações 


$$
x_{2}(t)=\sum_{k=-\infty}^{\infty} a_{2} g_{2}(t-k T)
$$

onde $g_{1}(t)$ e $g_{2}(t)$ são os pulsos básicos. agora desiguais e complexos. Pode-se mostrar que uma equalização mais eficiente é obtida para este tipo de sinal quando não se impõe a restrição de equalizadores iguais como os da Fig.1.

A equalização assimétrica foi analisada recentemente em [4] e [5]. Em [4] são obtidas expressões do erro médio quadrático minimo para diversas situações típicas que correspondem à assimetria nas componentes em fase e em quadratura. Em [5], assumindo o critério de mínimo erro médio quadrático. são deduzidas expressões gerais para as funções de transferência ótimas dos equalizadores assimétricos. supondo que estes têm um número infinito de coeficientes. As expressões gerais do erro médio quadrático correspondente são tambérn aí obtidas.

No presente trebalho, procuramos conpletar estes resultados analíticos deduzindo, para equalizadores assimétricos com número finito de derivações. as expressões dos coeficientes ótimos e do erro médio quadrático a partir das formas de onda dos pulsos básicos. Quanto aos resultados numéricos. além de compararmos o potencial de melhoria de equalizadores simétricos e assimétricos em presença de desvanecimento seletivo, situação não considerada em [4] e [5]. é feita também uma comparação da velocidade de convergência destes dois tipos de equalizadores.

\section{Otimização dos equalizadores as simétricos}

Vamos considerar a otimização dos equalizadores da Fig.1 de modo a minimizar o erro médio quadrático entre o símbolo transmitido e o sinal à saída do equalizador, definido como

$$
\varepsilon=E\left\{\left[\mathrm{e}_{1}(\mathrm{kT})\right]^{2\}}+\mathrm{E}_{\{}\left[\mathrm{e}_{2}(\mathrm{kT})\right]^{2\}}\right.
$$

onde,

$$
\begin{aligned}
& e_{1}(k T)=y_{1}(k T)-a_{1}, k \\
& e_{2}(k T)=y_{2}(k T)-a_{2, k}
\end{aligned}
$$


e. de acordo com a Fig.1

$$
\begin{aligned}
& y_{1}(k T)=\sum_{i=-N}^{N} w_{11, i} x_{1}[(k-i) T]+\sum_{i=-N}^{N} W_{12, i} x_{2}[(k-i) T] \\
& y_{2}(k T)=\sum_{i=-N}^{N} W_{22, i} x_{2}[(k-i) T]+\sum_{i=-N}^{N} W_{21, i} x_{1}[(k-i) T]
\end{aligned}
$$

Vamos utilizar o critério do erro médio quadrático mínimo para calcular os coeficientes dos equalizadores. Este cálculo é feito igualando-se a zero as derivadas parciais do erro médio quadrático em relação aos coeficientes, como mostrado no Apêndice A. Chega-se assim às seguintes expressões para os coeficientes ótimos

$$
\begin{aligned}
& \mathbf{W}_{11}=\left[\mathbf{R}_{\mathrm{x}_{2} \mathrm{x}_{1}}^{-1} \mathbf{R}_{\mathrm{x}_{1}}-\mathbf{R}_{\mathrm{x}_{2}}^{-1} \mathbf{R}_{\mathrm{x}_{1} \mathrm{x}_{2}}\right]^{-1}\left[\mathbf{R}_{\mathrm{x}_{2} \mathrm{x}_{1}}^{-1} \mathbf{R}_{\mathrm{a}_{1} \mathrm{x}_{1}}-\mathbf{R}_{\mathrm{x}_{2}}^{-1} \mathbf{R}_{\mathrm{a}_{1} \mathrm{x}_{2}}\right] \\
& \mathbf{W}_{12}=-\mathbf{R}_{\mathrm{x}_{2}}^{-1} \mathbf{R}_{\mathrm{x}_{1} \mathrm{X}_{2}} \mathbf{W}_{11}+\mathbf{R}_{\mathrm{x}_{2}}^{-1} \mathbf{R}_{\mathrm{a}_{1 \times 2}}
\end{aligned}
$$

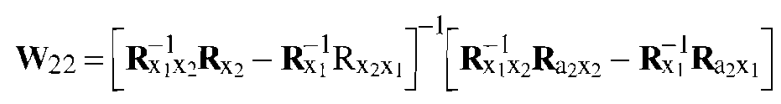

$$
\begin{aligned}
& \mathbf{W}_{21}=-\mathbf{R}_{\mathrm{x}_{1}}^{-1} \mathbf{R}_{\mathrm{x}_{2} x_{1}} \mathrm{~W}_{22}+\mathbf{R}_{\mathrm{x}_{1}}^{-1} \mathbf{R}_{\mathrm{a} 2 \mathrm{x}_{1}}
\end{aligned}
$$

onde $\mathbf{W}_{\mathrm{ij}}(\mathrm{i}, \mathrm{j}=1,2)$ é o vetor de coeficientes definido em (A13) e $\mathbf{R}_{\mathrm{x}_{\mathrm{j}}}, \mathbf{R}_{\mathrm{x}_{\mathrm{j}} \mathrm{x}_{\mathrm{j}}}$ e $\mathbf{R}_{\mathrm{a}_{\mathrm{i}} \mathrm{x}_{\mathrm{j}}}$ $(i, j=1,2)$, são matrizes definidas em (A11), (A12), e (A14) para $i=1$ e $j=2$.

As funções autocorrelação e correlação cruzada que aparecem em (A11), (A12) e (A14) podem ser expressas em função dos pulsos básicos $\mathrm{g}_{1}(\mathrm{t})$ e $\mathrm{g}_{2}(\mathrm{t})$ que aparecem em (5a) e (5b). Substituindo estas expressões em (A7) e (A8), assumindo que as amplitudes $\left\{a_{1, k\}}\right\}$ $\left\{\mathrm{a}_{2, \mathrm{k}}\right\}$ são variáveis aleatórias de médias nulas estatisticamente independentes entre si e com variâncias iguais a $\sigma^{2}$, chega-se, após algumas manipulações algébricas, às seguintes expressões

$$
\left.\mathrm{R}_{\mathrm{x}_{1}}(\mathrm{j} \mathrm{T})=\sigma^{2} \sum_{\mathrm{k}=-\infty}^{\infty} \operatorname{Re}\left[\mathrm{g}_{1}(\mathrm{kT})\right] \operatorname{Re}_{\{} \mathrm{g}_{1}[(\mathrm{k}+\mathrm{j}) \mathrm{T}]\right\}+
$$




$$
\begin{aligned}
& \mathrm{R}_{\mathrm{x}_{2}}(\mathrm{j} \mathrm{T})=\sigma^{2} \sum_{\mathrm{k}=-\infty}^{\infty} \operatorname{Im}\left[\mathrm{g}_{1}(\mathrm{kT})\right] \operatorname{Im}\left\{\mathrm{g}_{1}[(\mathrm{k}+\mathrm{j}) \mathrm{T}]\right\}+ \\
& +\operatorname{Re}\left[\mathrm{g}_{2}(\mathrm{kT})\right] \operatorname{Re}\left\{\mathrm{g}_{2}[(\mathrm{k}+\mathrm{j}) \mathrm{T}]\right\} \\
& \mathrm{R}_{\mathrm{x}_{1} \mathrm{X}_{2}}(\mathrm{j} T)=\sigma^{2} \sum_{\mathrm{k}=-\infty}^{\infty} \operatorname{Re}\left[\mathrm{g}_{1}(\mathrm{kT})\right] \operatorname{Im}\left\{\mathrm{g}_{1}[(\mathrm{k}+\mathrm{j}) \mathrm{T}]\right\}- \\
& -\operatorname{Im}\left[\mathrm{g}_{2}(\mathrm{kT})\right] \operatorname{Re}\left\{\mathrm{g}_{2}[(\mathrm{k}+\mathrm{j}) \mathrm{T}]\right\} \\
& R_{x_{1} x_{2}}(j T)=R_{x_{2} x_{1}}(-j T) \\
& \mathrm{R}_{\mathrm{a}_{1} \mathrm{x}_{1}}(\mathrm{j} T)=\sigma^{2} \operatorname{Re}\left[\mathrm{g}_{1}(\mathrm{j} \mathrm{T})\right] \\
& \mathrm{R}_{\mathrm{a}_{2} \mathrm{x}_{2}}(\mathrm{jT})=\sigma^{2} \operatorname{Re}\left[\mathrm{g}_{2}(\mathrm{j} \mathrm{T})\right] \\
& \mathrm{R}_{\mathrm{a}_{1} \mathrm{x}_{2}}(\mathrm{j} \mathrm{T})=\sigma^{2} \operatorname{Im}\left[\mathrm{g}_{1}(\mathrm{jT})\right] \\
& R_{\mathrm{a}_{2} \mathrm{x}_{1}}(\mathrm{j} T)=-\sigma^{2} \operatorname{Im}\left[\mathrm{g}_{2}(\mathrm{j} T)\right]
\end{aligned}
$$

(11a)

Pode-se verificar que se $g_{1}(t)=g_{2}(t)$

$$
\begin{gathered}
R_{x_{1}}(j T)=R_{x_{2}}(j T) \\
R_{x_{1} x_{2}}(j T)=-R_{x_{2} x_{1}}(j T) \\
R_{a_{1} x_{1}}(j T)=R_{a_{2} x_{2}}(j T) \\
R_{a_{1} x_{2}}(j T)=-R_{a_{2} x_{1}}(j T)
\end{gathered}
$$


Levando estas igualdades em (9), observa-se que o sistema de equações (9a) e (9b) é igual ao sistema formado por (9c) e (9d) e, neste caso, $\mathrm{W}_{11}=\mathrm{W}_{22} \mathrm{eW}_{12}=-\mathrm{W}_{21}$. Definindo-se então

$$
\mathbf{W}=\mathbf{W}_{11}+\mathbf{j} \mathbf{W}_{12}
$$

tem-se

$$
\begin{array}{r}
\mathbf{R}_{\mathrm{x}}=\mathbf{R}_{\mathrm{x}_{1}}+\mathbf{R}_{\mathrm{x}_{2}}+\mathrm{i}\left(\mathbf{R}_{\mathrm{x}_{1} \mathrm{x}_{2}}-\mathbf{R}_{\mathrm{x}_{2} \mathrm{x}_{1}}\right) \\
\mathbf{R}_{\mathrm{ax}}=\mathbf{R}_{\mathrm{a}_{1} \mathrm{x}_{1}}+\mathbf{R}_{\mathrm{a}_{2} \mathrm{x}_{2}}+\mathrm{j}\left(\mathbf{R}_{\mathrm{a}_{1} \mathrm{x}_{2}}+\mathbf{R}_{\mathrm{a}_{2} \mathrm{x}_{1}}\right)
\end{array}
$$

e resolvendo um dos dois sistemas chega-se a

$$
\mathbf{W}=\mathbf{R}_{\mathrm{x}}^{-1} \mathbf{R}_{\mathrm{ax}}
$$

que é a solução conhecida para os coeficientes ótimos no caso simétrico [1].

Em função dos coeficientes ótimos calculados através de (9) pode-se calcular o erro médio quadrático dado por (6). Para isto basta usar (7) e (8) em (6), e usar notação matricial para escrever

$$
\begin{aligned}
& \mathrm{e}_{1}(\mathrm{kT})^{2}= \mathbf{W}_{11}^{\mathrm{T}} \mathbf{R}_{\mathrm{x}_{1}} \mathbf{W}_{11}+\mathbf{W}_{12}^{\mathrm{T}} \mathbf{R}_{\mathrm{x}_{2}} \mathbf{W}_{12}+2 \mathbf{W}_{11}^{\mathrm{T}} \mathbf{R}_{\mathrm{x}_{2} \mathrm{x}_{1}} \mathbf{W}_{12}- \\
&-2 \mathbf{W}_{11}^{\mathrm{T}} \mathbf{R}_{\mathrm{a}_{1} \mathrm{x}_{1}}-2 \mathbf{W}_{12}^{\mathrm{T}} \mathbf{R}_{\mathrm{a}_{1} \mathrm{x}_{2}}+\sigma^{2} \\
& \mathrm{e}_{2}(\mathrm{kT})^{2}=\mathbf{W}_{22}^{\mathrm{T}} \mathbf{R}_{\mathrm{x}_{2}} \mathbf{W}_{22}+\mathbf{W}_{21}^{\mathrm{T}} \mathbf{R}_{\mathrm{x}_{1}} \mathbf{W}_{21}+2 \mathbf{W}_{22}^{\mathrm{T}} \mathbf{R}_{\mathrm{x}_{1} \mathrm{x}_{2}} \mathbf{W}_{21}- \\
&-2 \mathbf{W}_{22}^{\mathrm{T}} \mathbf{R}_{\mathrm{a}_{2} \mathrm{x}_{2}}-2 \mathbf{W}_{21}^{\mathrm{T}} \mathbf{R}_{\mathrm{a}_{2} \mathrm{x}_{1}}+\sigma^{2}
\end{aligned}
$$

Usando (9) e notando que

$$
\left[\mathbf{W}_{11}^{\mathrm{T}} \mathbf{R}_{\mathrm{x}_{2} \mathrm{x}_{1}} \mathbf{W}_{12}\right]=\left[\mathbf{W}_{11}^{\mathrm{T}} \mathbf{R}_{\mathrm{x}_{2} \mathrm{x}_{1}} \mathbf{W}_{\mathrm{l} 2}\right]^{\mathrm{T}}=\mathbf{W}_{12}^{\mathrm{T}} \mathbf{R}_{\mathrm{x}_{2} \mathrm{x}_{1}} \mathbf{W}_{11}=\mathbf{W}_{12}^{\mathrm{T}} \mathbf{R}_{\mathrm{x}_{1} \mathrm{x}_{2}} \mathbf{W}_{11}
$$

e, analogamente,

Revista da Sociedade Brasileira de Telecomunicações

Volume 7 - Número 1 - Dezembro 1992 


$$
\left[\mathbf{W}_{22}^{\mathrm{T}} \mathbf{R}_{\mathrm{x}_{1} \mathrm{X}_{2}} \mathbf{W}_{21}\right]=\mathbf{W}_{21}^{\mathrm{T}} \mathbf{R}_{\mathrm{x}_{2} \mathrm{X}_{1}} \mathbf{W}_{22}
$$

chega-se, finalmente, a

$$
\varepsilon^{2}=2 \sigma^{2}-\mathbf{W}_{11}^{\mathrm{T}} \mathbf{R}_{\mathrm{a}_{1} \mathrm{x}_{1}}-\mathbf{W}_{12}^{\mathrm{T}} \mathbf{R}_{\mathrm{a}_{1} \mathrm{X}_{2}}-\mathbf{W}_{22}^{\mathrm{T}} \mathbf{R}_{\mathrm{a}_{2} \mathrm{x}_{2}}-\mathbf{W}_{21}^{\mathrm{T}} \mathbf{R}_{\mathrm{i}_{2} \mathrm{x}_{1}}
$$

\section{Aplicação}

Estudos comparativos do desempenho de equalizadores transversais simétricos e assimétricos com 5 e 7 derivações foram realizados para diferentes casos de transmissão digital em um sistema com modulação QAM-64, taxa de transmissão de $144 \mathrm{Mbit} / \mathrm{s}$ e filtros de transmissão e recepção com função de transferência em raiz quadrada de cosseno levantado com roll-off 0,5. O sinal QAM-64, é transmitido através de um canal com função de transferência dada pelo modelo de dois raios [6] cujos parâmetros são 0,5 para amplitude do raio secundário, 6,3 ns de retardo entre o raio primário e o secundário, e desvio do notch igual a $10 \mathrm{MHz}$. O desempenho é medido pelo erro médio quadrático mínimo obtido com a expressão (21). Para obtenção do sinal QAM foi utilizado o programa ASTRAL [7].

Inicialmente consideramos uma situação em que os pulsos básicos são diferentes mas proporcionais, ou seja. $g_{2}(t)=\mathrm{kg}_{1}(t)$, onde $k$ é uma constante. Nas Figs. 3 e 4 são apresentadas curvas de erro médio quadrático versus a diferença normalizada de amplitudes, $\mathrm{d}=1-\mathrm{k}$. Em um outro conjunto de resultados, as duas formas do pulso básico $\mathrm{g}_{1}(\mathrm{t})$ e $\mathrm{g}_{2}(\mathrm{t})$ resultam de filtros formatadores desiguais na transmissão e na recepção. Supõe-se, que os dois filtros de um mesmo canal (em fase ou em quadratura) têm o mesmo valor de roll-off, mas este valor é diferente para os dois canais. Os resultados estão mostrados nas Figs. 5 e 6. As curvas foram obtidas tomando-se um valor igual a 0,5 para o roll-off do canal em fase e valores menores para o roll-off do canal em quadratura.

Observa-se nas Figs. 3 e 4 que o desempenho do equalizador assimétrico é indiscutivelmente melhor para o caso onde se tem pulsos básicos com diferentes amplitudes, o que resulta em diferentes níveis de potência nas componentes em fase e em quadratura. Nessa situação o equalizador assimétrico consegue manter o erro médio quadrático no mesmo nível apesar do crescente desbalanceamento gerado. Analisando nas Figs. 5 e 6 o comportamento em função da diferença de roll-off entre os filtros, nota-se novamente o melhor desempenho do equalizador assimétrico, embora, aqui, esse tipo de equalizador não consiga compensar grandes diferenças de roll-off.

Para se ter uma avaliação mais completa do desempenho do equalizador assimétrico, seria interessante comparar também sua rapidez de convergência com a do equalizador simétrico. Isto foi feito utilizando os algoritmos de forçagem a zero usados em [4]. Para a equalização simétrica o algoritmo é dado por 


$$
\begin{aligned}
& W_{11, i}^{k+1}=W_{11, i}^{k}-\alpha \operatorname{Sgn}\left[e_{1}(k T) a_{1, k-i}\right]+\operatorname{Sgn}\left[e_{2}(k T) a_{2, k-1}\right] \\
& W_{12, i}^{k+1}=W_{12, i}^{k}-\alpha \operatorname{Sgn}\left[e_{2}(k T) a_{1, k-i}\right]-\operatorname{Sgn}\left[e_{1}(k T) a_{2, k-i}\right]_{j}^{1}
\end{aligned}
$$

onde $W_{j, i}^{k}$ é o coeficiente de ordem i do equalizador $\mathrm{j}$ na iteração $\mathrm{k}, \mathrm{i}=-\mathrm{N},-\mathrm{N}+1, \ldots, \mathrm{N}, \mathrm{j}=$ $11,12,21$ e 22 e a função Sgn é dado por

$$
\operatorname{Sgn}(x)=\left\{\begin{array}{cc}
1 & x \geq 0 \\
0 & x=0 \\
-1 & x=<0
\end{array}\right.
$$

Note-se que, na equalização simétrica, $\mathbf{W}_{11}=\mathbf{W}_{22}$ e $\mathbf{W}_{21}=-\mathbf{W}_{12}$. Para a equalização assimétrica tem-se

$$
\begin{aligned}
& W_{11, i}^{k+1}=W_{11, i}^{k}-\alpha \operatorname{Sgn}\left[e_{1}(k T) a_{1, k-i}\right] \\
& W_{12, i}^{k+1}=W_{12, i}^{k}-\alpha \operatorname{Sgn}\left[e_{1}(k T) a_{2, k-i}\right] \\
& W_{21, i}^{k+1}=W_{21, i}^{k}-\alpha \operatorname{Sgn}\left[e_{2}(k T) a_{1 . k-i}\right] \\
& W_{22, i}^{k+1}=W_{22, i}^{k}-\alpha \operatorname{Sgn}\left[e_{2}(k T) a_{2 . k-i}\right]
\end{aligned}
$$

Para comparar a convergência do equalizador assimétrico com a do simétrico, a operação destes equalizadores com os algoritmos dados por (22) e (24) foi simulada com o programa ASTRAL obtendo-se o resultado mostrado na Fig. 7 para equalizadores com 7 derivações. O sinal utilizado nesta comparação é um sinal QAM-64 com simetria nos canais em fase e em quadratura com taxa de bit igual a $144 \mathrm{Mbit} / \mathrm{s}$ e filtros de transmissão e recepção em raiz quadrada de cosseno levantado com roll-off: igual a 0,35 . Observa-se na Fig.7 que, efetivamente, o tempo de convergência do equalizador assimétrico é aproximadamente o dobro do tempo gasto na equalização simétrica.

\section{Conclusĩ̃o}

Neste trabalho foram desenvolvidas expressões analíticas para os coeficientes ótimos, segundo o critério do mínimo erro médio quadrático, de equalizadores assimétricos de comprimento finito, a partir das formas de onda dos pulsos básicos de um sinal QAM. Os

Revista da Sociedade Brasileira de Telecomunicações 
resultados representam uma generalização de expressões semelhantes, já bem conhecidas para equalizadores simétricos, e incluem ainda expressões do erro médio quadrático mínimo correspondente.

As expressões analíticas desenvolvidas foram utilizadas para comparar, através do erro médio quadrático, o potencial de melhoria das equalizações simétrica e assimétrica em um sistema QAM sujeito a desvanecimento seletivo. Além disto, através de simulação, foi feita uma comparação da convergência dos dois tipos de equalizadores.

Os resultados numéricos confirmaram a maior capacidade da equalização assimétrica em corrigir distorções, como já verificado em trabalhos anteriores. Por outro lado, a convergência dos equalizadores assimétricos se mostrou mais lenta do que a dos equalizadores simétricos em uma situação onde não há assimetria entre as componentes em fase e em quadratura do sinal QAM.

Conclui-se, portanto, do presente estudo e de outros trabalhos, que é interessante, em princípio, usar equalizadores assimétricos, sobretudo em sistemas mais sensíveis a desbalanceamento como sistemas QAM com grande número de níveis (QAM-64, QAM128 etc.). Equalizadores assimétricos consegurem corrigir estes desbalanceamentos com maior eficiência. Quando o sistema é balanceado mostrou-se, por outro lado, que o equalizador assimétrico se reduz ao equalizador simétrico, apresentando portanto o mesmo desempenho.

Sob o ponto de vista prático pode não ser interessante utilizar equalização assimétrica quando se deseja, sobretudo, obter uma rápida convergência do algoritmo de adaptação. Para os sistemas rádio digitais, no entanto, esta desvantagem parece não ser muito importante quando comparada às vantagens, já mencionadas, da equalização assimétrica.

Um último comentário, ainda com relação aos aspectos de implementação, é que na equalização em banda básica a equalização assimétrica não implica em nenhum aumento de complexidade. Pelo contrário, a formação do sinal de controle é mais simples do que na equalização simétrica. Na implementação em FI, entretanto, ocorre um substancial aumento de complexidade na implementação na medida em que a assimetria implica em passar de dois para quatro o número de equalizadores.

\section{Apêndice A}

Neste apêndice são obtidas as expressões dos coeficientes ótimos de um conjunto de equalizadores assimétricos, de comprimento finito, a partir das funções autocorrelação e correlação cruzada do sinal digital. Para isto, basta igualar a zero as derivadas parciais do erro médio quadrático, dado por (6), em relação aos coeficientes. Fazendo isto para o coeficiente $W_{11, j}$ tem-se

$$
\frac{\partial \varepsilon}{\partial W_{11, j}}=\frac{\partial}{\partial W_{11, j}} E\left\{\left[e_{1}(k T)\right]^{2}\right\}=
$$




$$
\begin{gathered}
=E\left\{2 e_{1}(k T) \frac{\partial}{\partial W_{11, j}} \sum_{i=-N}^{N} W_{11, i} x_{1}[(k-i) T]\right\}= \\
=2 R_{e_{i} x_{l}}(-j T)=0 ; j=-N, \ldots, N
\end{gathered}
$$

onde.

$$
\mathrm{R}_{\mathrm{e}_{1} \mathrm{X}_{1}}(-\mathrm{j} \mathrm{T})=2 \mathrm{E}\left\{\mathrm{e}_{1}(\mathrm{kT}) \mathrm{x}_{1}[(\mathrm{k}-\mathrm{j}) \mathrm{T}]\right\}
$$

Analogamente

$$
\begin{array}{ll}
\frac{\partial \varepsilon}{\partial W_{21, j}}=2 R_{e_{1} x_{2}}(-j T)=0 & j=-N, \ldots, N \\
\frac{\partial \varepsilon}{\partial W_{12, j}}=2 R_{e_{2} x_{1}}(-j T)=0 & j=-N, \ldots . N \\
\frac{\partial \varepsilon}{\partial W_{22, j}}=2 R_{e_{2} x_{2}}(-j T)=0 & j=-N, \ldots, N
\end{array}
$$

Desenvolvendo-se (A1) tem-se

$$
\begin{gathered}
E\left\{\left[\sum_{i=-N}^{N} \mathrm{~W}_{11, \mathrm{i}} \mathrm{x}_{1}[(\mathrm{k}-\mathrm{i}) \mathrm{T}]+\sum_{\mathrm{i}=-\mathrm{N}}^{\mathrm{N}} \mathrm{W}_{12, \mathrm{i}} \mathrm{x}_{2}[(\mathrm{k}-\mathrm{i}) \mathrm{T}]-\mathrm{a}_{1, \mathrm{k}}\right] \mathrm{x}_{1}[(\mathrm{k}-\mathrm{j}) \mathrm{T}]\right\}= \\
=\sum_{\mathrm{i}=-\mathrm{N}}^{N} \mathrm{~W}_{11, \mathrm{i}} \mathrm{E}\left\{\mathrm{x}_{1}[(\mathrm{k}-\mathrm{i}) \mathrm{T}] \mathrm{x}_{1}[(\mathrm{k}-\mathrm{j}) \mathrm{T}]\right\}+ \\
\left.+\sum_{\mathrm{i}=-\mathrm{N}}^{\mathrm{N}} \mathrm{W}_{12, \mathrm{i}} \mathrm{E}_{[2} \mathrm{x}_{2}[(\mathrm{k}-\mathrm{i}) \mathrm{T}] \mathrm{x}_{1}[(\mathrm{k}-\mathrm{j}) \mathrm{T}]\right\}-\mathrm{E}\left\{\mathrm{a}_{1, \mathrm{k}} \mathrm{x}_{1}[(\mathrm{k}-\mathrm{j}) \mathrm{T}]=0\right.
\end{gathered}
$$

Definindo

$$
\mathrm{R}_{\mathrm{x}_{\mathrm{i}}}(\tau)=\mathrm{E}\left[\mathrm{x}_{\mathrm{i}}(\mathrm{t}) \mathrm{x}_{\mathrm{i}}(\mathrm{t}+\tau)\right]
$$

Revista da Sociedade Brasileira de Telecomunicações 


$$
\mathrm{R}_{\mathrm{X}_{\mathrm{i}} \mathrm{X}_{\mathrm{j}}}(\tau)=\mathrm{E}\left[\mathrm{x}_{\mathrm{i}}(\mathrm{t}) \mathrm{x}_{\mathrm{j}}(\mathrm{t}+\tau)\right]
$$

tem-se, equivalentemente

$$
\begin{gathered}
\sum_{i=-N}^{N} W_{11, j R_{x_{1}}}[(i-j) T]+\sum_{i=-N}^{N} W_{12, i} R_{x_{2} x_{1}}[(i-j) T]-R_{a_{1} x_{1}}(-j T)=0 \\
j=-N, \ldots . N
\end{gathered}
$$

Em notação matricial, a equação (A9) pode ser reduzida a

$$
\mathbf{R}_{\mathrm{x}_{1}} \mathbf{W}_{11}+\mathbf{R}_{\mathrm{x}_{2} \mathrm{x}_{1}} \mathbf{W}_{12}=\mathbf{R}_{\mathrm{a}_{1} \mathrm{x}}
$$

onde

$$
\begin{aligned}
& \mathbf{R}_{\mathrm{X}_{1}}=\left[\begin{array}{cccc}
\mathrm{R}_{\mathrm{x}_{1}}(0) & \mathrm{R}_{\mathrm{X}_{1}}(\mathrm{~T}) & \ldots & \mathrm{R}_{\mathrm{X}_{1}}(2 \mathrm{NT}) \\
\mathrm{R}_{\mathrm{X}_{1}}(-\mathrm{T}) & \mathrm{R}_{\mathrm{x}_{1}}(0) & \ldots & \mathrm{R}_{\mathrm{X}_{1}}[(2 \mathrm{~N}-1) \mathrm{T}] \\
\cdot & \cdot & \cdot & \cdot \\
\cdot & \cdot & \cdot & \cdot \\
\cdot & \cdot & \cdot & \cdot \\
\mathrm{R}_{\mathrm{X}_{1}}(-2 \mathrm{NT}) & \ldots & \ldots & \mathrm{R}_{\mathrm{X}_{1}}(0)
\end{array}\right] \\
& \mathbf{R}_{\mathrm{x}_{2} \mathrm{x}_{1}}=\left[\begin{array}{cccc}
\mathrm{R}_{\mathrm{x}_{2} \mathrm{x}_{1}}(0) & \mathrm{R}_{\mathrm{x}_{2} \mathrm{x}_{1}}(\mathrm{~T}) & \ldots & \mathrm{R}_{\mathrm{x}_{2} \mathrm{x}_{1}}(2 \mathrm{NT}) \\
\mathrm{R}_{\mathrm{x}_{2} \mathrm{x}_{1}}(-\mathrm{T}) & \mathrm{R}_{\mathrm{x}_{2} \mathrm{x}_{1}}(0) & \ldots & \mathrm{R}_{\mathrm{x}_{2} \mathrm{x}_{1}}[(2 \mathrm{~N}-1) \mathrm{T}] \\
\cdot & \cdot & \cdot & \cdot \\
\cdot & \cdot & \cdot & \cdot \\
\cdot & \cdot & \cdot & \cdot \\
\mathrm{R}_{\mathrm{x}_{2} \mathrm{x}_{1}}(-2 \mathrm{NT}) & \ldots & \ldots & \mathrm{R}_{\mathrm{x}_{2} \mathrm{x}_{1}}(0)
\end{array}\right]
\end{aligned}
$$

(A11) 


$$
\begin{gathered}
\mathbf{W}_{\mathrm{mn}}=\left[\begin{array}{c}
\mathrm{W}_{\mathrm{mn},-\mathrm{N}} \\
\mathrm{W}_{\mathrm{mn},-\mathrm{N}+1} \\
\cdot \\
\cdot \\
\mathrm{W}_{\mathrm{mn}, \mathrm{N}}
\end{array}\right] \\
\mathbf{R}_{\mathrm{a}_{1} \mathrm{x}_{1}}=\left[\begin{array}{c}
\mathrm{R}_{\mathrm{a}_{1} \mathrm{x}_{1}}[(\mathrm{NT})] \\
\mathrm{R}_{\mathrm{a}_{1} \mathrm{x}_{1}[(\mathrm{~N}-1) \mathrm{T}]} \\
\cdot \\
\cdot \\
\mathrm{R}_{\mathrm{a}_{1} \mathrm{X}_{1}}[-(\mathrm{NT})]
\end{array}\right]
\end{gathered}
$$

Estendendo o mesmo desenvolvimento a (A3), (A4) e (A5) obtém-se o seguinte sistema de equações

$$
\begin{aligned}
& \mathbf{R}_{\mathrm{x}_{1}} \mathbf{W}_{11}+\mathbf{R}_{\mathrm{x}_{2} \mathrm{X}_{1}} \mathbf{W}_{12}=\mathbf{R}_{\mathrm{a}_{1} \mathrm{x}_{1}} \\
& \mathbf{R}_{\mathrm{x}_{1} \mathrm{X}_{2}} \mathbf{W}_{11}+\mathbf{R}_{\mathrm{x}_{2}} \mathbf{W}_{12}=\mathbf{R}_{\mathrm{a}_{1} \mathrm{X}_{2}} \\
& \mathbf{R}_{\mathrm{x}_{2} \mathrm{x}_{1}} \mathbf{W}_{22}+\mathbf{R}_{\mathrm{x}_{1}} \mathbf{W}_{21}=\mathbf{R}_{\mathrm{a}_{2} \mathrm{x}_{1}} \\
& \mathbf{R}_{\mathrm{x}_{2}} \mathbf{W}_{22}+\mathbf{R}_{\mathrm{x}_{1} \mathrm{x}_{2}} \mathbf{W}_{21}=\mathbf{R}_{\mathrm{a}_{2} \mathrm{X}_{2}}
\end{aligned}
$$

Resolvendo este sistema de equações obtém-se as expressões dadas por (9a) a (9d).

\section{Referências}

[1] S. Benedetto, E. Biglieri, V. Castellani, "Digital Transmission Theory", PrenticeHall Inc., 1987.

[2] N. Amitay, L. Greenstein, "Multipath outage performance of digital radio receivers using finite-tap adaptive equalizers", IEEE Trans. on Comm., Vol. COM-32, $\mathrm{N}^{\circ} 5$, May 1984, pp 597-608.

[3] Kurt Metzger, "Comparison of different transversal equalization strategies for multilevel digital radio systems", Proc. of 1987 SBMO International Symposium, Rio de Janeiro, Julho de 1987, pp 1197-1202.

Revista da Sociedade Brasileira de Telecomunicações

Volume 7 - Número 1 - Dezembro 1992 
[4] H. Sari, G. Karam, "Compensation of Modem Imperfections by Adaptive Filtering", Proc. of Globecom-86, pp 15.1.1-15.1.6.

[5] H. Sari, G. Karam, “Asymetric Baseband Equalization”, IEEE Trans. on Communications, Vol. 36, N9, Sept 1988, pp 1073-1078.

[6] W.D. Rummler, "A New Selective Fading Model: Application to Propagation Data”, Bell System Tech. Journal, Vol 58, pp 1037-1071, May-June 1979.

[7] J.C. Brandão, "Análise e Simulação de Transmissão Digital em Microcomputador:, Anais do $7^{\circ}$ Simpósio Brasileiro de Telecomunicações, Florianópolis, Setembro de 1989 , pp 338-343.

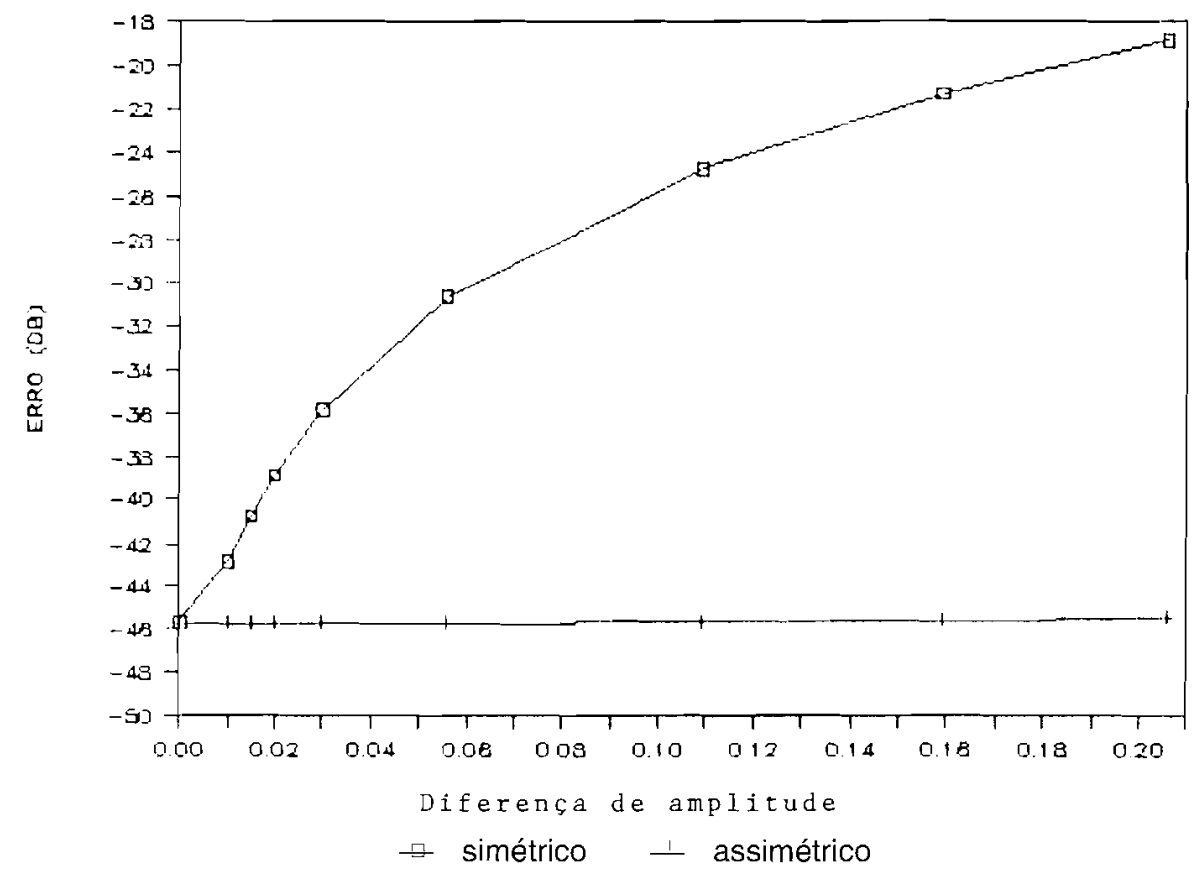

Figura 3 - Variação do erro médio quadrático mínimo com a diferença de amplitude entre os pulsos de banda básica de um sistema QAM-64 sujeito a desvanecimento seletivo (equalizadores de 5 derivações). 


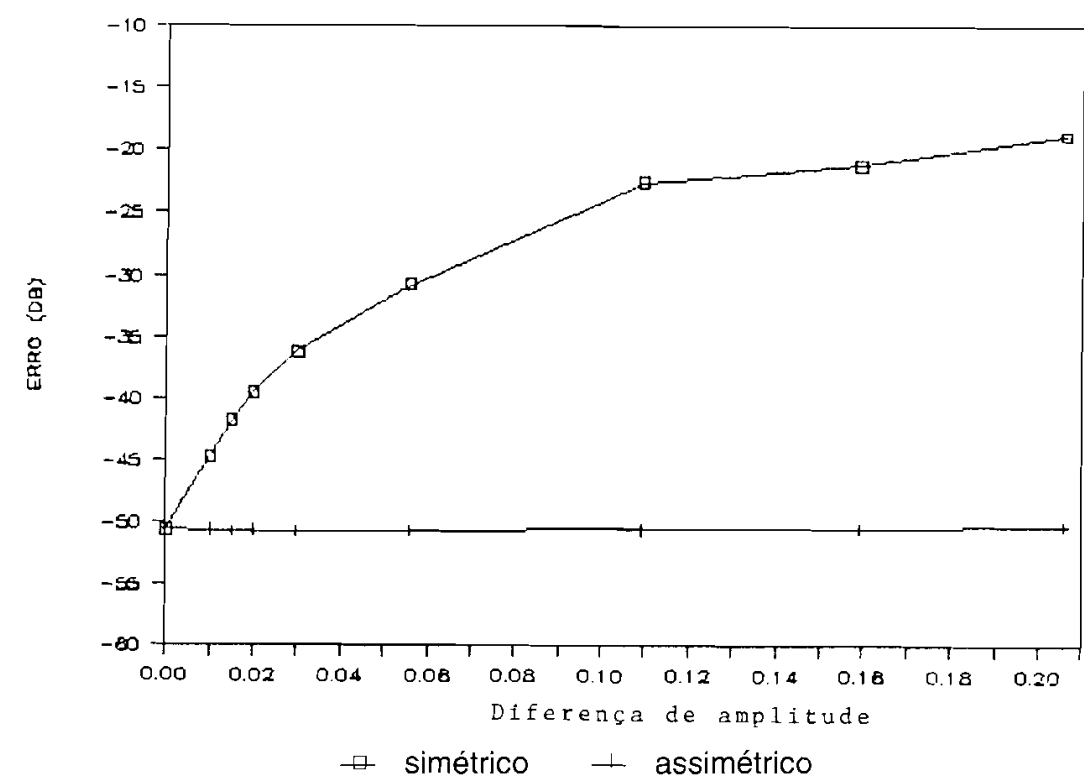

Figura 4 - Variação do erro médio quadrático mínimo com a diferença de amplitude entre os pulsos de banda básica de um sistema QAM-64 sujeito a desvanecimento seletivo (equalizadores de 7 derivações).

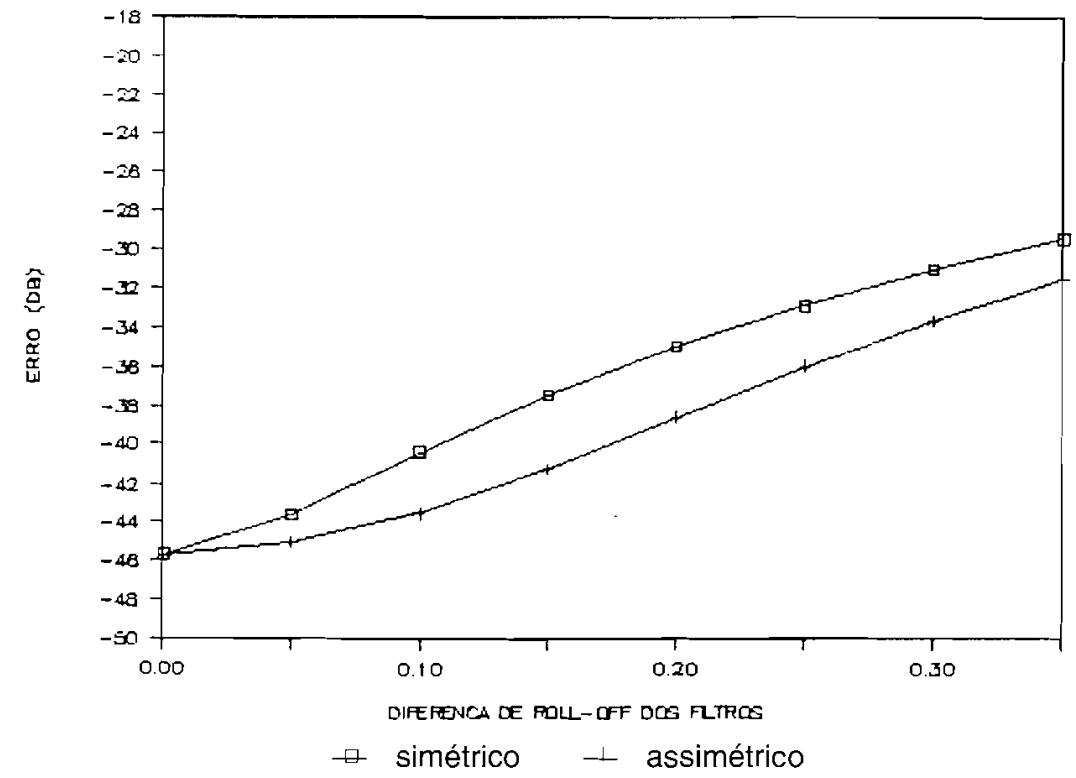

Figura 5 - Variação do erro médio quadrático mínimo com a diferença entre os valores de "roll-off" dos filtros nos canais em fase e em quadratura de um sistema QAM64 sujeito a desvanecimento seletivo (equalizadores com 5 derivações).

Revista da Sociedade Brasileira de Telecomunicações 


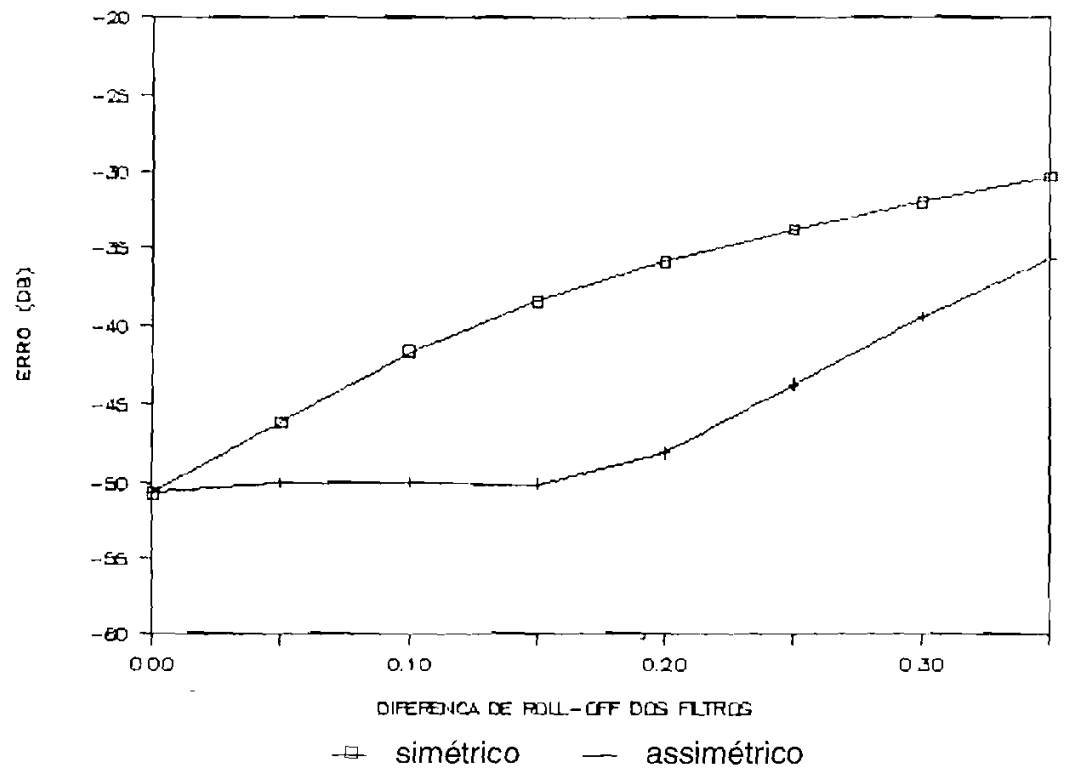

Figura 6 - Variação do erro médio quadrático mínimo com a diferença entre os valores de "roll-off" dos filtros nos canais em fase e em quadratura de um sistema QAM64 sujeito a desvanecimento seletivo (equalizadores com 7 derivações).

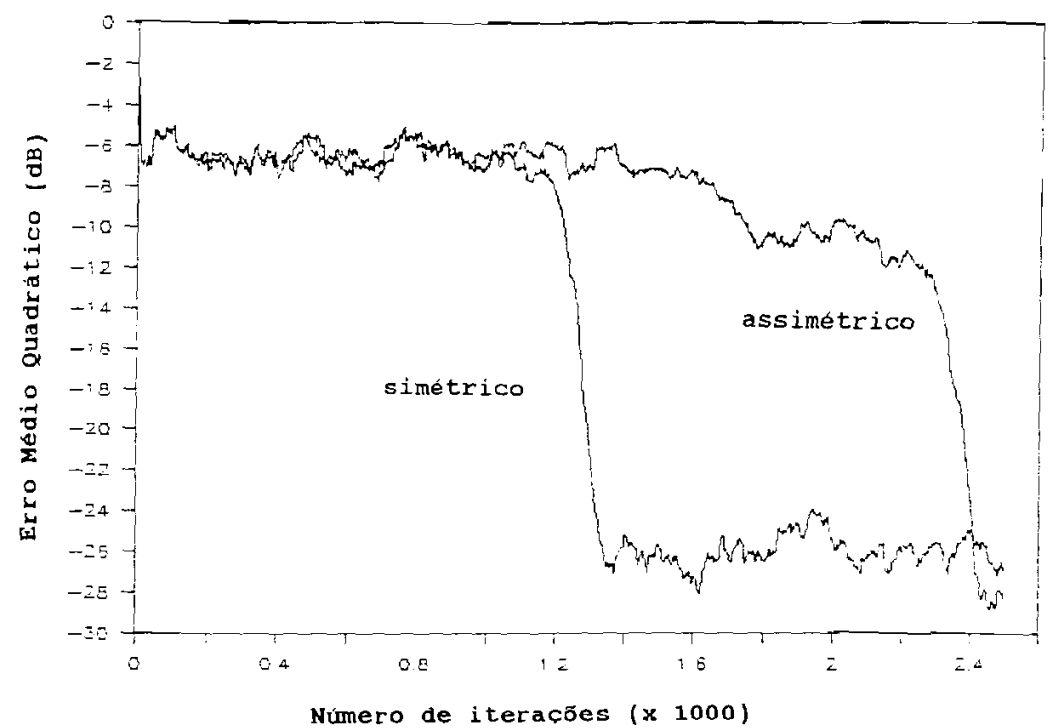

Figura 7 - Convergência dos equalizadores simétricos e assimétricos em um sistema QAM-64 sujeito a desvanecimento seletivo. 


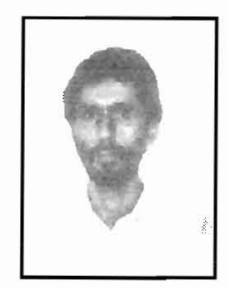

João Célio Barros Brandão - Engenheiro Eletricista pela Universidade Federal de Juiz de Fora (1969) e Mestre em Ciências em Engenharia Elétrica pela PUC do Rio de Janeiro (1973). Professor e pesquisador do CETUC - Centro de Estudos em Telecomunicações da PUC-RIO - desde 1971, onde foi vice-diretor (1979-81) e é atualmente Professor Associado e coordenador do Grupo de Sistemas de Telecomunicações. Em 1987 e 1988 trabalhou no COMSAT LABORATORIES, U.S.A., na área de Sistemas de Comunicações via Satélite. No CETTJC tem participado como pesquisador e coordenador de diversos projetos de pesquisa na área de interferência entre sistemas de comunicações e transmissão digital, em convênio com a TELEBRÁS. Ao longo destes projetos supervisionou o desenvolvimento do software ASTRAL para análise e simulaçao de sistemas de transmissão digital que tem sido usado para ensino e pesquisa na PUC-RIO no CPqD-TELEBRÁS e em outras instituições. É membro da Comissao Brasileira de Estudos em Rádio Comunicações do Ministério das Comunicações. É sócio fundador da Sociedade Brasileira de Telecomunicações onde tem colaborado na organização de simpósios e atualmente é o Editor do Boletim Informativo.

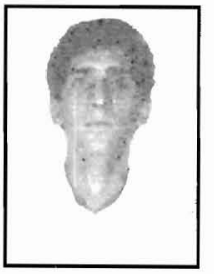

Álvaro Luis Guimarães de Brito - Formado em Engenharia Elétrica, com ênfase em eletrônica pela Pontifícia Universidade Católica do Rio de Janeiro (PUC/RIO) no ano de 1991. Como estagiário trabalhou no Centro de Estudos em Telecomunicações da Universidade Católica (CETUC), em simulação e análise por software de transmissão digital. Atualmente é funcionário da Empresa Moddata S.A. Engenharia de Telecomunicações e Informática, trabalhando na área de projeto e instalação de sistemas de rádio digital de média capacidade, na faixa de SHF. 